\title{
Maîtrise du comptage de l'eau sur les conduites de gros diamètre «Le cas de Paris »
}

\author{
A. Tiret \\ Compagnie des Eaux de Paris
}

J. Stevance

Parisienne des Eaux

\section{Le service de l'eau à Paris: une longue histoire}

\section{I. Des porteurs d'eau aux pompes à feu}

Pendant toute la période allant de l'antiquité jusqu'à la fin de l'ancien régime, en passant par le Moyen-Age, il n'existe pas de service public de distribution de l'eau à Paris. En dehors de quelques privilégiés, abbayes ou palais royaux qui disposent d'adductions particulières d'eau de source, la majorité des Parisiens doivent s'alimenter en eau, soit à partir de puits particuliers, soit en allant à la fontaine publique; la confrérie des porteurs d'eau dont l'apogée se situe sous le règne d'Henri IV, où ils sont plus de 5000 , assure à cette époque une distribution d'eau à domicile, pour les Parisiens les plus aisés.

Le même Henri IV, conscient des lacunes du système et du manque de ressources en eau fait installer les premières machines hydrauliques remontant l'eau de la Seine à la Samaritaine et au Pont Notre-Dame. Celles-ci sont trop sujettes aux aléas du gel et de la sécheresse pour garantir la pérennité de l'approvisionnement.

A la fin du $x^{\prime} \|^{\mathrm{c}}$ siècle, Paris compte un million d'habitants, la production d'eau disponible ne dépasse guère $3000 \mathrm{~m}^{3}$ par jour, la Seine demeurant la principale ressource, dont la mauvaise qualité de l'eau provoque des épidémies.

\subsection{La compagnie des eaux de Paris}

Avec la concession accordée aux Frères PÉRIER en 1777 et la naissance de la première Compagnie des Eaux de Paris en 1778 , le service de l'eau dans la capitale rentre dans l'ère moderne. La création de réservoirs et la mise en place des premières pompes à feu de Chaillot et du Gros-Caillou permettent d'améliorer la situation : les Parisiens disposent par jour de $5500 \mathrm{~m}^{3}$ d'eau potable supplémentaires. Grâce aux pompes à feu, l'élévation des eaux de la Seine n'est plus influencée par les conditions climatiques et la régularité de l'approvisionnement est assurée.

La Compagnie des Eaux de Paris gère le premier vrai Service Public de l'eau. Elle met en place les premières conduites desservant les particuliers. Les privilèges de certains concessionnaires privés disparaissent: ils sont désormais soumis au même régime d'approvisionnement en eau que l'ensemble des Parisiens.

Les porteurs d'eau voient leur activité fortement concurrencée par cette modernisation de la distribution. Ils trouvent en MirABEAU un ardent et efficace défenseur; Beaumarchais soutient la Compagnie des Eaux de Paris.

Malheureusement, les Banques n'ayant pas soutenu suffisamment les Frères PÉRIER, la Compagnie des Eaux de Paris (première du nom) disparaît en 1780 à l'issue d'une existence éphémère et non sans avoir constitué le premier service public de distribution d'eau à Paris.

A cette même date, la Ville de Paris prend la maîtrise de toutes les eaux distribuées, réunissant en une seule administration les eaux dites « du Roy » et celles dites « de la Ville». L'administration municipale continue néanmoins à faire appel aux entreprises spécialisées pour assurer le Service Public de l'Eau.

\section{Control and metering of water on large diameter conduits : "The Case of Paris »}

After a historical recall on the establishing of water public utility in Paris and on present device for water supplying in the chief town, the authors bring forward the circumstances of the 1985 privatization and the consequences of the division into two water management districts, each one confided to a private company. They highlight the establishing of the counting device for invoicing. 


\subsection{Les travaux d'HaussmanN et de BELGRAND}

A partir du Second Empire, la mise en place d'un Service des Eaux efficace et l'extension des limites de Paris vont permettre de doter la capitale d'un système d'alimentation en eau moderne et de qualité.

C'est à cette période que pour assurer l'approvisionnement en eau des Parisiens, le Préfet Haussmann, aidé de I'Ingénieur BELGRAND, fait déclarer d'utilité publique la dérivation des eaux de la Dhuis et de la Vanne, sources d'eau souterraine captées à une centaine de kilomètres de Paris. Aux deux aqueducs construits à cet effet s'ajoutent plus tard ceux de l'Avre (1895), du Loing (1900) et de la Voulzie (1925).

Pour éviter que se renouvellent les épidémies d'origine hydrique des années 1830 , des procédés de filtration lente sont mis en place à la fin du $\mathrm{XIX}^{\mathrm{e}}$ siècle pour les eaux de la Seine à Ivry et de la Marne à Saint-Maur.

C'est sous le « règne » du Préfet Haussmann que sont entrepris les travaux de mise en place d'un système d'assainissement à Paris, tout à fait exemplaire, puisque plus de cent quarante ans après, ce même réseau d'égout assure toujours efficacement la collecte des eaux usées de la capitale et continue de jouer le rôle de galerie technique qu'HaUSSMANN avait envisagé dès l'origine.

C'est ainsi que près de $95 \%$ du linéaire des canalisations d'eau de la capitale chemine à l'intérieur des galeries d'égout, ce qui constitue un cas de figure tout à fait exceptionnel. Egalement exceptionnelle, la présence sous les voies de Paris d'un double réseau de distribution d'eau, l'un véhiculant l'eau potable alimentant les Parisiens et l'autre transportant de l'eau de Seine pour des utilisations d'eau non potable, comme le nettoyage des caniveaux.

Eugène BELGRAND aura été le concepteur et le réalisateur des principaux ouvrages structurants du service de distri- bution d'eau de Paris et la plupart de ceux-ci constituent encore aujourd'hui l'ossature de l'approvisionnement en eau de la capitale.

\subsection{La situation actuelle}

Aujourd'hui, les 2 millions de Parisiens, ainsi que les 4 millions de Banlieusards qui viennent chaque jour travailler dans la capitale génèrent des besoins en eau potable qui représentent en moyenne plus de $750000 \mathrm{~m}^{3}$ par jour, soit près de 270 millions de $\mathrm{m}^{3}$ chaque année.

Pour satisfaire ces besoins, il est fait appel d'une part, par priorité aux ressources souterraines mises en valeur par Eugène BELgRAND et acheminées jusqu'à Paris par des aqueducs, la plupart du temps gravitaires et d'autre part, aux eaux de surface traitées. La proportion entre chacune des deux origines varie d'une année sur l'autre, mais plus de la moitié de l'eau distribuée à Paris, provient du soussol, ce qui représente une situation exceptionnelle comparée à celle des autres grandes métropoles dans le monde.

1.4.1. Les eaux d'origine souterraine proviennent de captages situés entre 80 et $150 \mathrm{~km}$ de Paris. L'aqueduc de l'Avre (région de Dreux) peut produire jusqu'à $160000 \mathrm{~m}^{3}$ par jour, les aqueducs de la Vanne, de la Voulzie et du Loing (régions de Sens, Provins et Fontainebleau), $360000 \mathrm{~m}^{3}$ par jour. Soit, pour l'ensemble des sources, un total de $520000 \mathrm{~m}^{3}$ par jour, au maximum.

1.4.2. Les eaux de surface, prélevées dans la Seine et la Marne, sont traitées sur trois sites. Les usines d'Ivry et d'Orly sur la Seine ont chacune une capacité de $300000 \mathrm{~m}^{3}$. L'usine de Joinville sur la Marne, actuelle-

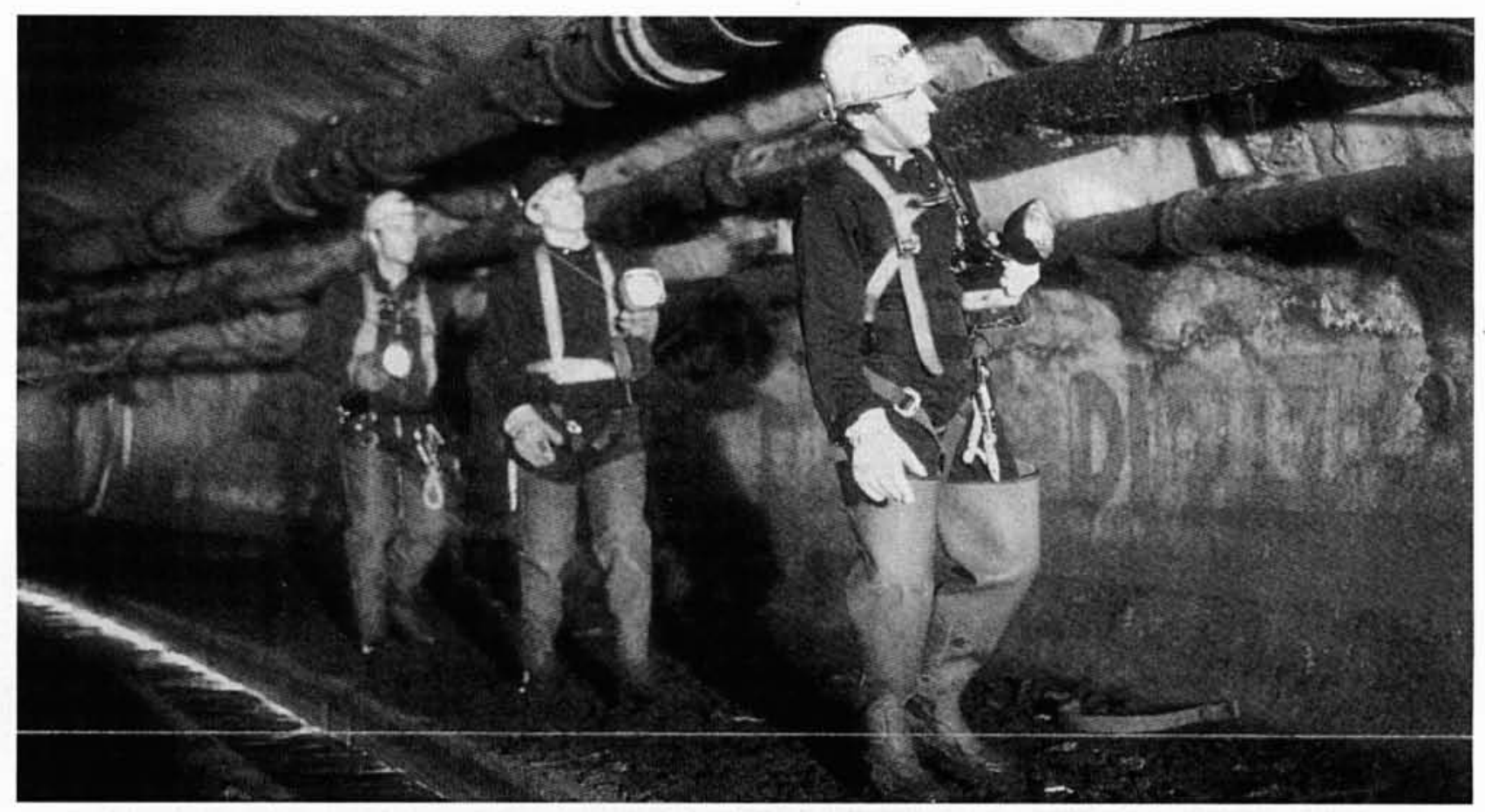

\section{Visite des canalisations.}


ment en cours de reconstruction, peut produire $210000 \mathrm{~m}^{3}$ par jour. Soit un total maximum de $810000 \mathrm{~m}^{3}$ par jour.

Depuis les aqueducs et les usines, l'eau est acheminée dans cinq grands réservoirs situés soit en périphérie de Paris (Saint-Cloud, L'Haÿ-les-Roses), soit dans Paris intramuros (Montsouris, Les Lilas et Ménilmontant). Ils ont une capacité globale de stockage d'environ $1200000 \mathrm{~m}^{3}$, représentant plus d'une journée et demie de consommation.

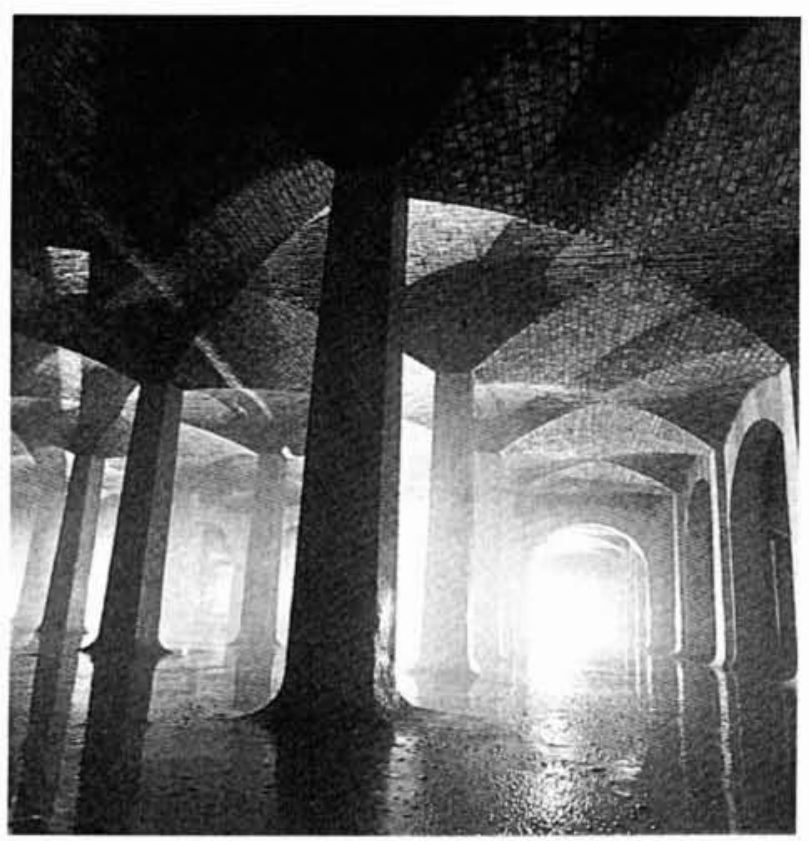

2. Le réservoir de Ménilmontant.

A partir de ces réservoirs, les eaux sont réparties entre deux réseaux d'élévations différentes, pour atteindre le robinet du Parisien :

- le réseau bas, qui correspond à la zone centrale de Paris, permet de distribuer l'eau dans les quartiers situés à moins de 40 mètres d'altitude. Alimenté à partir du réservoir de Montsouris, situé sur la rive gauche (cote altimétrique d'environ 75 mètres NGF), il distribue environ $250000 \mathrm{~m}^{3}$ par jour ;

- le réseau haut, en périphérie de Paris, alimente des secteurs situés entre 40 et 60 mètres d'altitude et distribue environ $500000 \mathrm{~m}^{3}$ par jour depuis les quatre autres réservoirs.

A ces deux réseaux principaux s'ajoutent les réseaux particuliers situés dans les secteurs de Paris qui ne peuvent être alimentés directement par le réseau haut. Il s'agit pour l'essentiel des quartiers de Belleville, Montmartre, Passy et Montsouris, situés à plus de 60 mètres d'altitude (130 mètres pour Montmartre et Belleville) et qui disposent également de réservoirs de stockage de capacité moindre, mais dont la cote altimétrique est suffisante pour assurer une pression normale sur ces Buttes.

\section{La privatisation du service de l'eau}

\subsection{Les acteurs de la privatisation}

A la fin de l'année 1984, la Ville de Paris décide de déléguer son service de distribution d'eau, tout en gardant la maitrise de ses moyens de production. Elle confie celuici à deux sociétés privées: La Compagnie des Eaux de Paris d'une part, filiale de la Générale des Eaux, pour la distribution d'eau sur les 14 arrondissements de la rive droite, et la Parisienne des Eaux, filiale de la Lyonnaise des Eaux-Dumez, pour la distribution d'eau sur les 6 arrondissements de la rive gauche.

A ce stade, les motivations qui animent la ville sont multiples, mais l'une d'entre elles justifie à elle seule sa décision. Il s'agit de lancer un programme ambitieux de renouvellement des conduites de distribution d'eau dont la plupart sont plus que centenaires, afin de réduire le taux de pertes en eau du réseau, qui atteignait à cette époque près du quart de la production totale d'eau potable de la capitale. Les $1800 \mathrm{~km}$ de canalisations d'eau potable et les $1600 \mathrm{~km}$ de conduites d'eau non potable ont, de fait, besoin d'une action de rénovation en profondeur nécessitant des moyens financiers importants, que les deux sociétés s'engagent à mobiliser.

Deux années plus tard, en janvier 1987, la production d'eau est concédée à la SAGEP, Société d'économie mixte, dans laquelle la Ville de Paris est majoritaire, la Générale des Eaux et la Lyonnaise des Eaux-Dumez siégeant chacune à part égale aux côtés de l'actionnaire majoritaire.

Ses fonctions principales consistent à produire l'eau pour l'ensemble des Parisiens, à l'acheminer jusqu'à l'entrée de Paris intra-muros et la vendre en gros aux deux gestionnaires de la distribution. La SAGEP assure aussi pour le compte de la Ville de Paris le contrôle des contrats des deux gestionnaires de la distribution.

\subsection{Les contrats et leur mode de fonctionnement}

Pour tenir compte des programmes importants de rénovation auxquels s'étaient obligés tant les gestionnaires pour la distribution, que la SAGEP pour la production, l'ensemble des contrats passés avec la Ville ont été conclus pour une période de vingt-cinq années.

Dans ces contrats, trois phases d'application successives ont été prévues dès l'origine pour ce qui concerne les moyens de détermination de la facturation de l'eau livrée en gros par la SAGEP à chaque gestionnaire.

\section{Phase 1}

Lors de cette phase initiale qui a démarré avant même 1987, puisqu'en 1985 et 1986 la Ville assurait elle-même la production d'eau, les volumes fournis aux gestionnaires étaient déterminés à partir des moyens de comptage préexistants et répartis entre eux deux, au prorata des ventes d'eau aux abonnés de chacune des rives. Ces moyens de comptage étaient fort rustiques. En effet, ils n'avaient qu'un rôle d'indicateur et non de compteur, du fait de l'unicité du gestionnaire avant 1985. La plupart du temps, ce n'étaient même pas des débitmètres, mais des compteurs horaires mesurant le temps de fonctionnement des pompes de refoulement, quand il y avait des pompes. 
Quant il n'y avait pas de pompes (cas des aqueducs), des organes déprimogènes simples jouaient le rôle de points de mesure. Ces points de mesure de débit, qu'ils soient de lecture directe (comptages) ou obtenus par une autre méthode, étaient tous situés au départ de la production, sur les usines ou sur les sources, et non à l'arrivée sur Paris.

\section{Phase 2 : Installation de compteurs sur l'ensemble de la péri- phérie de Paris}

Dès 1987, une vingtaine de débitmètres à ultrasons ont été placés par la SAGEP sur les différentes adductions, à leur point de pénétration dans Paris intra-muros. Ces appareils étaient installés pour compter l'eau au moment où elle rentrait physiquement dans Paris, en rive droite, comme en rive gauche.

Le passage de la phase 1 à la phase 2 s'est fait grâce à une méthode identifiée sous le terme générique de «Loi de Mariotte ", puisqu'elle est basée sur le principe que $\boldsymbol{P V}=$ constante dans laquelle :

$P=$ Prix du mètre cube livré en gros

$V=$ Volume livré en gros.

Il était convenu qu'en passant d'un système de comptage à l'autre, l'ancien prix au $\mathrm{m}^{3}$ multiplié par le volume fourni sur la période précédant la prise en compte des compteurs périphériques devait être égal au nouveau prix du $\mathrm{m}^{3}$ multiplié par le volume mesuré par le nouveau système de comptage, sur la même période de référence. L'écart pour passer d'un régime à l'autre était de l'ordre de $3 \%$, sur la base des années 1987 et 1988 .

Cela conduisit à corriger le prix de fourniture de l'eau en gros à partir du $1^{\text {er }}$ janvier 1989 , du fait du changement de système de comptage et de sa prise en compte à cette même date pour la facturation.

\section{Phase 3 : Phase définitive - Installation de compteurs entre les deux rives}

De la même façon que pour la périphérie, une vingtaine de débitmètres à ultrasons furent installés sur les différentes conduites situées sur les ponts de Paris, pour mesurer les volumes transférés entre les deux rives. Les contrats prévoyaient que le $1^{\text {er }}$ janvier de l'année suivant la constatation par les gestionnaires et la SAGEP du bon fonctionnement de ces compteurs (ceux qui séparaient physiquement la rive droite et la rive gauche) les volumes fournis à chaque gestionnaire seraient calculés à partir des indications de ces compteurs, et non plus au prorata du volume vendu aux abonnés de chacune des rives, comme dans les phases 1 et 2 .

\subsection{Les résultats}

Au terme de huit années d'efforts déployés par la Compagnie des Eaux de Paris et la Parisienne des Eaux, pour la rénovation du réseau de distribution d'eau qui leur avait été confiée par la Ville de Paris, des résultats très significatifs ont d'ores et déjà pu être enregistrés. En effet, le taux de pertes en eau du réseau est passé sur cette période de $24 \%$ à $10 \%$, ce qui représente en valeur absolue une économie de l'ordre de 35 millions de $\mathrm{m}^{3} /$ jour sur la base des consommations actuelles du réseau Parisien; soit l'équivalent de la production annuelle d'une unité de
$100000 \mathrm{~m}^{3} /$ jour de capacité ou de la consommation d'une agglomération de 400000 habitants...

\section{Le dispositif de comptage de l'eau}

\subsection{Les capteurs de débit}

Pour l'ensemble des comptages périphériques, c'est-à-dire ceux qui comptent l'eau livrée par la SAGEP aux deux gestionnaires, une vingtaine de compteurs de diamètre 2500 à $800 \mathrm{~mm}$ ont été mis en place, dont :

— trois électromagnétiques,

- un ultrason bicorde (c'est en fait le compteur le plus important qui compte l'eau de l'usine d'Orly rentrant en rive gauche),

- 16 ultrasons monocordes.

Pour les comptages de transfert, la quasi-totalité des points de comptage ont été équipés de débitmètres ultrasons monocordes (diamètres compris entre 1500 et $400 \mathrm{~mm})$.

Il faut savoir qu'à l'origine ces ultrasons monocordes ont été choisis pour deux raisons :

La première est une raison financière. A l'époque, en 1987 , le coût d'installation du comptage à ultrasons était relativement moins élevé que celui du comptage électromagnétique.

La deuxième résidait dans la facilité d'installation, puisqu'il y avait au total 20 compteurs sur les périphériques, 18 compteurs sur les transferts, ce qui faisait au total 38 compteurs à installer dans un minimum de temps et avec le moins de contraintes possible, notamment en matière de modifications hydrauliques.

A l'époque, la décision avait été prise d'installer ces compteurs à ultrasons sur l'ensemble des sites, sauf dans certains cas où la géométrie était vraiment très défavorable et où l'électromagnétique s'imposait donc.

\subsection{Les flux à mesurer}

Le tableau ci-dessous donne les chiffres de ce qui a été produit sur Paris pour l'année 1992.

\begin{tabular}{|l|c|r|r|}
\hline (en milliers de $\mathrm{m}^{3}$ ) & $\begin{array}{c}\text { Rive } \\
\text { droite }\end{array}$ & $\begin{array}{c}\text { Rive } \\
\text { gauche }\end{array}$ & $\begin{array}{l}\text { TOTAL } \\
\text { PARIS }\end{array}$ \\
\hline $\begin{array}{l}\text { Volumes introduits } \\
\text { (par SAGEP) }\end{array}$ & 72100 & 199300 & 271400 \\
\hline $\begin{array}{l}\text { Volumes mis en } \\
\text { distribution par rive }\end{array}$ & 185500 & 85900 & 271400 \\
\hline $\begin{array}{l}\text { Volumes transférés } \\
\text { (sens } R G \rightarrow R D) \\
\text { positif }\end{array}$ & +113400 & -113400 & \\
\hline
\end{tabular}

Sur cette seule année, la totalité des volumes livrés sur Paris représente un peu moins de 272 millions de $\mathrm{m}^{3}$. La 
livraison en rive gauche, c'est-à-dire l'eau qui rentre au sud de Paris représente près de 200 millions de $\mathrm{m}^{3}$, donc une grande partie de ce qu'utilise I'ensemble de Paris.

En rive droite, un peu plus de 70 millions de $\mathrm{m}^{3}$ ont été livrés, d'où un volume transféré de rive gauche en rive droite extrêmement important, puisqu'il représentait en 1992 plus de 110 millions de $\mathrm{m}^{3}$.

Se déduisant des chiffres ci-dessus, les volumes mis en distribution sur la rive droite s'élèvent à 185 millions de $\mathrm{m}^{3}$, et ceux mis en distribution sur la rive gauche représentent 86 millions de $\mathrm{m}^{3}$.

\subsection{L'expertise du dispositif}

Globalement, on peut dire qu'un quart de l'approvisionnement en eau de Paris arrive par le nord en rive droite, trois quarts rentrent par le sud en rive gauche et le transit entre la rive gauche et la rive droite de la production excédentaire sur les ponts de Paris, par l'intermédiaire de 18 points de comptage, représente $40 \%$ de l'approvisionnement global. Pour un prix d'achat, qui en 1992 avoisinait un peu plus de $2 \mathrm{~F}$ par $\mathrm{m}^{3}$, une erreur de $1 \%$ sur les volumes transférés par les ponts de Paris, représentait plus de 2 millions de francs par an. C'est dire que la précision demandée au système de comptage de l'eau devait être extrêmement importante et qu'aucune source d'erreur ne pouvait être tolérée.

C'est pourquoi la Compagnie des Eaux de Paris et la Parisienne des Eaux, avec l'aval de la SAGEP et de la Ville de Paris, ont décidé de recourir à une procédure d'expertise des dispositifs de comptage qui devaient servir de base à la répartition entre eux des charges de fourniture d'eau en gros, préalablement à leur prise en compte définitive.

Le choix de l'expert s'est fait par accord entre CEP et SPE et Monsieur Georges MAURIN, président de la Société Hydrotechnique de France, a accepté cette mission dès le mois de décembre 1991. Il s'est adjoint le concours de Monsieur ANDRÉ, spécialiste de l'hydraulique et des problèmes de macro-comptage, expérience acquise lors de sa carrière au sein des services de l'EDF.

L'expertise a pu être achevée le $1^{\text {er }}$ juillet 1992 en ce qui concerne les compteurs de transfert et a permis d'adopter le système de facturation prévu en phase 3 , dès l'exercice 1992, puis elle s'est prolongée jusqu'en novembre 1993 par une expertise des compteurs de périphérie.

Le déroulement de ces deux opérations, les moyens qui ont été mis en œuvre, ainsi que les conclusions qui ont été faites à l'issue de celles-ci, font l'objet des communications suivantes. 